Int. J. Dev. Biol. 49: 797-805 (2005)

doi: $10.1387 /$ ijdb.051976nt

Original Article

\title{
Fibroblast growth factor signalling and regional specification of the pharyngeal ectoderm
}

\author{
NINA TROKOVIC, RAS TROKOVIC and JUHA PARTANEN* \\ Institute of Biotechnology, Viikki Biocenter, University of Helsinki, Finland
}

\begin{abstract}
Branchial arch development involves dynamic interactions between neural crest cells as well as ectodermal, endodermal and mesodermal cell populations. Despite their importance and evolutionary conservation, the intercellular interactions guiding the early development of the branchial arches are still poorly understood. We have here studied fibroblast growth factor (FGF) signalling in early pharyngeal development. In mice homozygous for a hypomorphic allele of Fgfr1, neural crest cells migrating from the hindbrain mostly fail to enter the second branchial arch. This defect is non-cell-autonomous suggesting that Fgfr 1 provides a permissive environment for neural crest cell migration. Here we demonstrate localized down-regulation of the expression of the FGF responsive gene, Sprouty1 in the epithelium covering the presumptive second branchial arch of hypomorphic Fgfr1 mutants. This appears to result in a failure to establish an ectodermal signalling center expressing Fgf3 and Fgf15. We also studied differentiation of the ectoderm in the second branchial arch region. Development of the geniculate placode as well as the VIlth cranial ganglion is affected in Fgfr 1 hypomorphs. Our results suggest that Fgfr1 is important for localized signalling in the pharyngeal ectoderm and consequently for normal tissue interactions in the developing second branchial arch.
\end{abstract}

KEY WORDS: FGF signalling, fgfr1, patterning, mouse embryo, branchial arch, epibranchial placode, cranial nerve

\section{Introduction}

Six pairs of branchial arches form around pharyngeal foregut of developing mouse embryos. These bud like structures contain a core of paraxial mesoderm and aortic arch artery, which are surrounded by neural crest cells. The arches are covered with surface ectoderm from outside and pharyngeal endoderm from inside. Ectodermal pharyngeal clefts and endodermal pharyngeal pouches separate branchial arches in areas where ectodermal and endodermal cells are in direct contact.

Branchial arches arise in antero-posterior order between 8-11 days of embryonic development in the mouse, after which their cell types rearrange and further differentiate into terminal structures of head and neck regions. Neural crest cells form skeletal and connective structures, cranial paraxial mesoderm forms craniofacial muscles and endothelium, endoderm forms pharyngeal and middle ear epithelium and glandular structures while ectoderm forms epidermis and external acoustic meatus. Ectoderm also forms thickenings, called epibranchial placodes, which give rise to some of the sensory neurons of the cranial ganglia (Kaufman and Bard, 1999). Each arch also has its own identity and contributes to specific structures. For example, the second branchial arch contributes to the stapes of the middle ear, the styloid process of the temporal bone, the lesser horns of the hyoid bone, the facial nerve, muscles of facial expression and the stapedial artery.

Majority of earlier studies have highlighted the importance of the neural crest in the craniofacial patterning (Noden, 1983, 1988). Cranial neural crest cells, which originate from the dorsal part of the posterior midbrain and rhombomeres of the hindbrain, migrate ventrally in three distinct streams toward the branchial arches (Lumsden et al., 1991, Serbedzija et al., 1992). Early migrating neural crest cells populate the arches while late migrating crest cells form sensory nerve ganglia. Neural crest cells interact with other tissues and were thought to be the major player coordinating the integration of tissues into specific structures (Noden, 1983, Kontges and Lumsden, 1996). It was suggested that the segmental pattern of the hindbrain, encoded by Hox genes, is transmitted by neural crest cells to the branchial arches and cranial ganglia (Hunt etal., 1991). However, more recent data indicated that the initial development of these structures does not

Abbreviations used in this paper: fgf, fibroblast growth factor; fgfr, fibroblast growth factor receptor.

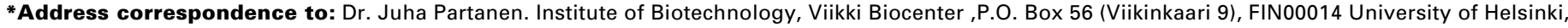
Finland. Fax: +358-9-1915-9366. e-mail: juha.m.partanen@helsinki.fi
} 
require neural crest cells (Veitch et al., 1999, Gavalas et al., 2001). Furthermore, there is now strong evidence that neural crest cells themselves are not irreversibly pre-patterned but are responsive to cues from their environment (Golding et al., 2000, Trainor and Krumlauf, 2000, 2001, Graham and Smith, 2001, Schilling et al., 2001, Couly et al., 2002, Ruhin, 2003).

What are then the tissues and signals responsible for patterning of the pharyngeal region? Cranial paraxial mesoderm is the other mesenchymal cell type of the branchial arches. It fills the core of the arch and differentiates into muscular structures (Meier and Tam, 1982). On its migratory route toward brancial arches, paraxial mesoderm is co-distributed with the neural crest cells originating at the same level along the anteroposterior axis. Studies on the craniofacial muscles indicated that this tissue functions passively in the head patterning, receiving instructive signals from the neural tube and the neural crest (Noden, 1983, 1986, Trainor et al., 1994, Kontges and Lumsden, 1996). However, more recent studies demonstrated that the paraxial mesoderm is a source of permissive signals important for maintaining expression of Hox genes in the neural crest (Trainor and Krumlauf, 2000) and suggested that it could be involved in regulation of the neural crest cell migration (Trainor et al., 2002a).

Recent studies have indicated importance of the endoderm in the pharyngeal patterning. Localized invaginations of the pharyngeal endoderm at sites between presumptive branchial arches, form segmentally organised pharyngeal pouches. They are important for directing the neural crest cell streams into separate branchial arches (Piotrowski and Nusslein-Volhard, 2000) as well as for induction of the epibranchial placodes (Begbie et al., 1999). Furthermore, recent analysis in chick (Couly et al., 2002) demonstrated that distinct stripes of the foregut endoderm send differential instructive signals to skeletogenic neural crest cells along the antero-posterior axis. Same study revealed that this antero-posterior regionalization of the foregut endoderm exists already at a stage before formation of the pharyngeal pouches.

Much less is known about early developmental function of the cranial surface ectoderm. Its inductive role was reported only at the later stage of the craniofacial development, during skeletal differentiation of the neural crest cells (Bee and Thorogood, 1980, Tucker and Sharpe, 1999). During embryonic development, cranial surface ectoderm forms antero-posterior series of the pharyngeal clefts and the epibranchial placodes. Already prior to formation of these segmentally organized structures, cranial ectoderm becomes regionalised into discrete domains known as ectomeres (Couly and Le Douarin, 1990). Although their function is not defined, distinct ectomeres coincide with the underlying streams of the migratory neural crest cells. Along this line, signaling from the surface ectoderm was suggested to be involved in regulation of the neural crest cell migration (Golding et al., 2002, Trainor et al., 2002a).

Growth factors of Wnt, Fgf and Tgf- $\beta$ families as well as Shh are signalling molecules which are expressed at different stages of the pharyngeal development. The fibroblast growth factors (FGFs) are encoded by 22 Fgf gene family members in the mouse. Numerous studies showed that FGFs are repeatedly involved in instructive signaling during embryonic develop-
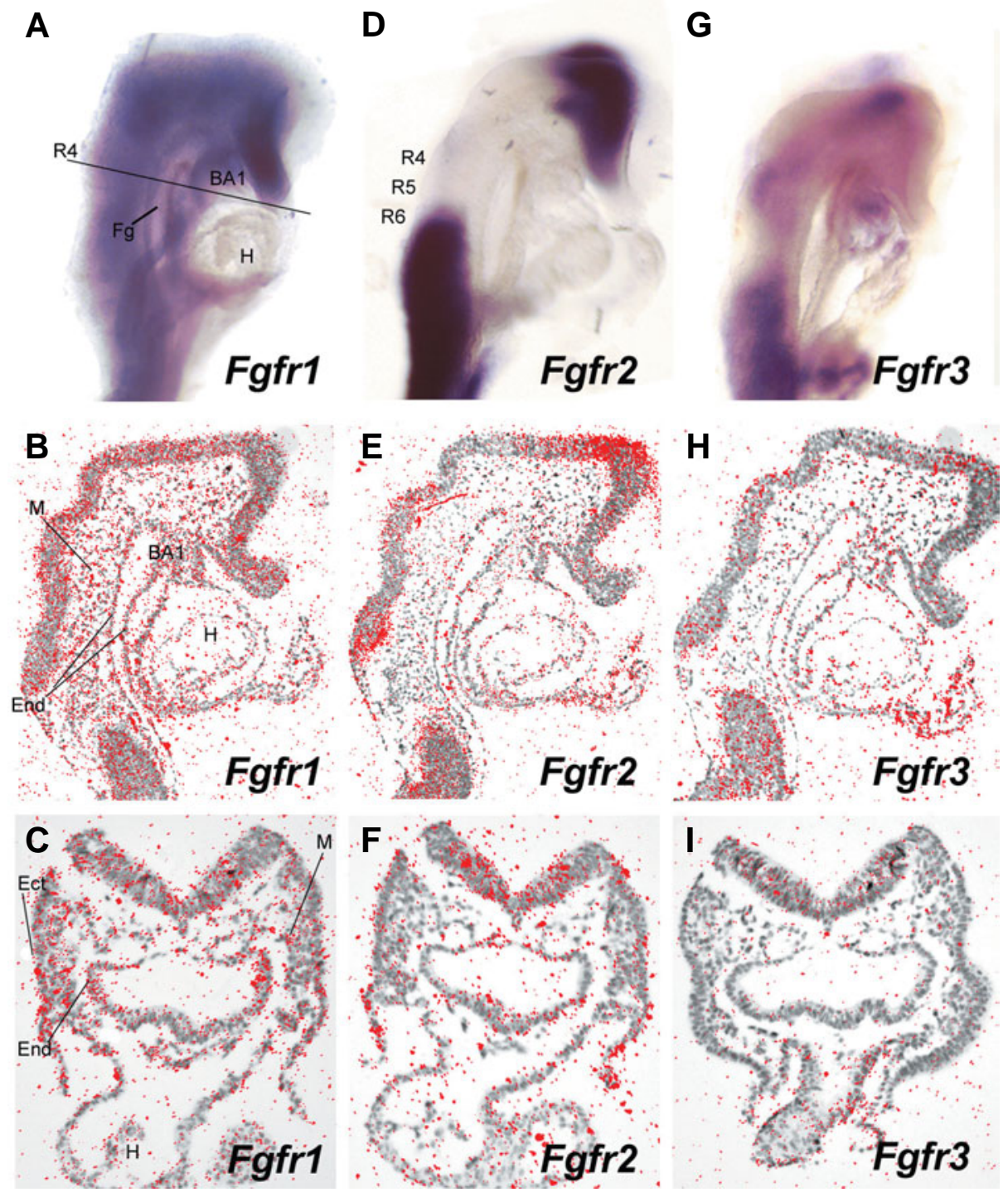

Fig. 1. Expression patterns of Fgfr1, Fgfr2 and Fgfr3 in the pharyngeal region of 8-somite stage mouse embryos. Whole mount in situ hybridization with Fgfr1 -3 probes (A,D,G). Level of transverse sections through the presumptive second arch $(\mathbf{C}, \mathbf{F}, \mathbf{I})$ is indicated in $(A)$. Fgfr1 $(\mathbf{A}, \mathbf{B}, \mathbf{C})$ is expressed throughout the pharyngeal region. Fgfr2 (D,E,F) is co-expressed with Fgfr1 at a low level. At the same time Fgfr3 $(\mathbf{G}, \mathbf{H}, \mathbf{I})$ appears to be expressed at a low level throughout the first branchial arch, but absent from the pharyngeal region posterior to it. BA1, first branchial arch; Ect, ectoderm; End, endoderm; Fg, foregut; $H$, heart; $M$, mesenchyme; R4, rhombomere 4. 

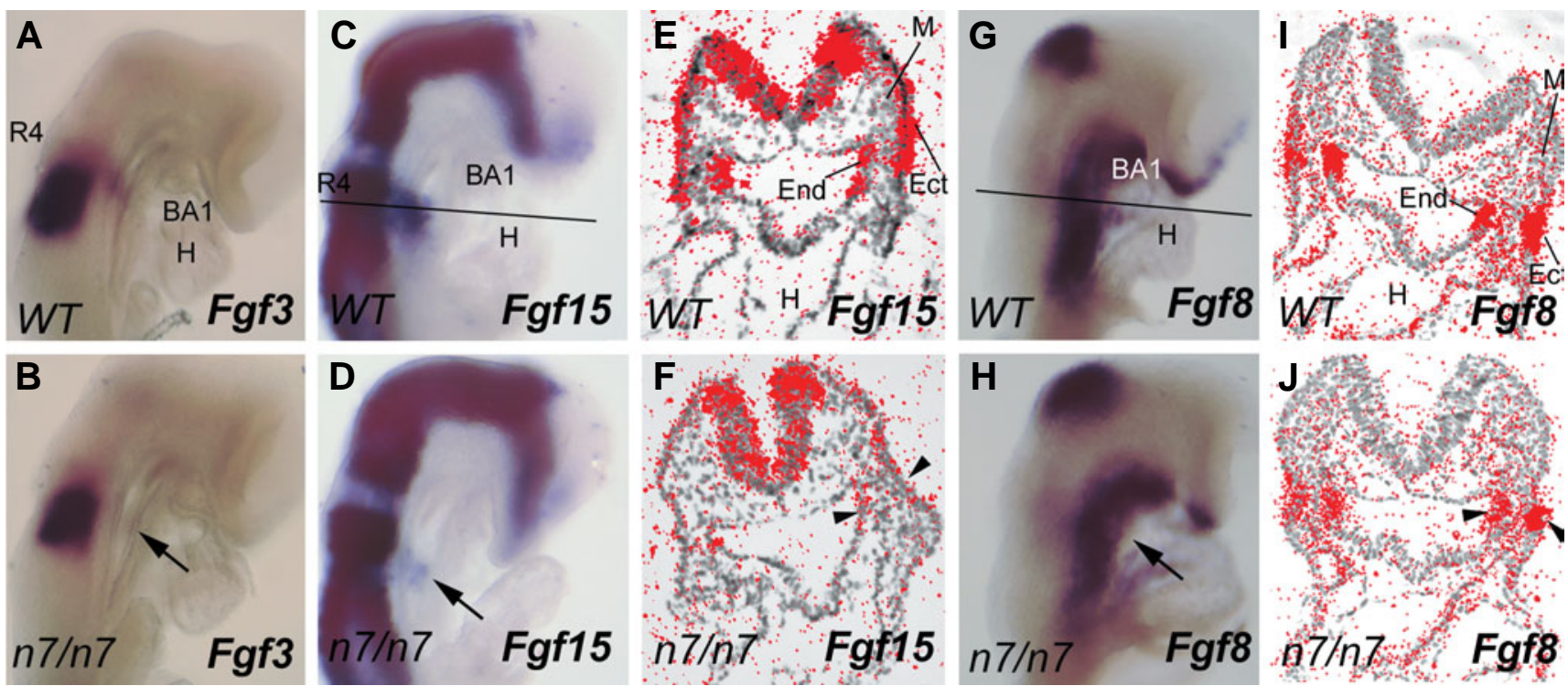

Fig. 2. Fgf3, Fgf15 and Fgf8 expression in the pharyngeal region of wild-type and Fgfr $\mathbf{1}^{\mathbf{n} 7 / \mathbf{n 7}}$ embryos at 8-9-somite stage. As shown by wholemount in situ hybridization, normal expression of Fgf3 (A) and Fgf15 (C) in the pharyngeal region is restricted to the presumptive second branchial arch region. Note that this domain of Fgf3 and Fgf15 expression is down-regulated in Fgfr 1 ${ }^{\mathrm{n} 7 / \mathrm{n} 7}$ embryos; see arrows in (B,D). Detection of Fgf15 by radioactive in situ hybridization on transverse sections through the presumptive second arch (level of sections is indicated in (C)) of wild-type (E) and mutant embryo (F). Note that Fgf15 expression in Fgfr $1^{\mathrm{n} 7 / \mathrm{n} 7}$ embryos is affected in both endoderm and ectoderm of the presumptive second arch, indicated by arrowheads in (F). Similar expression of Fgf8, in a broad area of the pharyngeal epithelium is detected in both control (G,I) and mutant embryos (H, J). $B A 1$, first branchial arch; Ect, ectoderm; End, endoderm; H, heart; $M$, mesenchyme; R4-6, rhombomere 4-6.

ment, for instance in regional specification and patterning of the hindbrain (Marin and Charnay, 2000, Waskiewicz et al., 2002) and branchial arch area (Tucker and Sharpe, 1999, Shigetani et al., 2000, Trainor et al., 2002b, David et al., 2002).

The effects of FGFs are mediated by four tyrosine kinasetype receptors, FGFR1-FGFR4. Fgfr1 null mutants are unable to gastrulate normally and die early during gestation (Deng et al., 1994, Yamaguchi et al., 1994). However, mice homozygous for a hypomorphic (partial loss-of-function) Fgfr 1 allele survive till birth (Partanen et al., 1998). Analysis of hypomorphic Fgfr1 mutants revealed defects in formation of the second branchial arch and in skeletal structures deriving from the first and second arch neural crest. We demonstrated that in hypomorphic Fgfr1 mutants neural crest cells migrating from rhombomere four level mostly fail to enter the second branchial arch. This neural crest migration defect is non-cell-autonomous and thus Fgfr 1 appears to be necessary for development of a permissive environment for neural crest cell migration into the second branchial arch (Trokovic et al., 2003a).

Here we further define molecular and cellular nature of the branchial arch defect in hypomorphic Fgfr1 mutants. We confirm our previous finding that Fgfr 1 is needed to establish appropriate patterns of gene expression in the cranial ectoderm overlying the presumptive second branchial arch and show that differentiation of this domain is affected in hypomorphic Fgfr 1 mutants. Localized expression patterns of Fgf3 and Fgf15 imply existence of a putative signalling centre in the surface ectoderm of the second branchial arch. Together, our results suggest that FGFR1 regulates establishment of this signalling center, which is required for normal integration and differentiation of the second branchial arch cell types.

\section{Results}

\section{Expression of Fgf-s, Fgfr-s and FGF responsive genes in the pharyngeal region}

To get insight into $F g f$ signalling in the pharyngeal region, we first analysed expression of receptors Fgfr1-3, ligands Fgf3, FgfB, Fgf15, Fgf17 and Fgf18 and downstream targets Spry1 and Spry4 in the developing branchial arches. We focused our studies on mouse embryos at 7-9-somite stage, the time point prior to appearance of morphological defect in the second branchial arch of Fgfr1 hypomorphs. For summary of the results, see Table 1.

TABLE 1

\begin{tabular}{|c|c|c|c|c|c|c|}
\hline \multicolumn{7}{|c|}{$\begin{array}{l}\text { EXPRESSION OF FGFs, FGFRs AND FGF RESPONSIVE } \\
\text { GENES IN THE PHARYNGEAL REGION AT E8.5 }\end{array}$} \\
\hline \multicolumn{3}{|c|}{ BA1 } & \multicolumn{4}{|c|}{ BA2 } \\
\hline Gene & M & End & Ect & $\mathbf{M}$ & End & Ect \\
\hline$\overline{F g f r 1}$ & ++ & ++ & ++ & ++ & ++ & $\overline{++}$ \\
\hline Fgfr2 & + & + & + & + & + & + \\
\hline Fgfr3 & + & + & + & - & - & - \\
\hline Fgf $^{*}$ & - & - & - & - & ++ & ++ \\
\hline Fgf8 & - & ++ & ++ & - & ++ & ++ \\
\hline Fgf15 & - & - & - & - & ++ & ++ \\
\hline Fgf17 & - & - & - & - & - & - \\
\hline Fgf18 & - & - & - & - & - & - \\
\hline Spry 1 & ++ & ++ & ++ & ++ & ++ & ++ \\
\hline Spry4 & ++ & ++ & - & ++ & ++ & - \\
\hline
\end{tabular}

${ }^{*}$ Fgf3 signal appears in endoderm shortly after expression in ectoderm.

+, low expression signal; ++, strong expression signal; -, absent expression signal; BA1, first branchial arch; BA2, second branchial arch; Ect, ectoderm; End, endoderm; M, mesenchyme. 
We found that Fgfr1 is broadly expressed in the pharyngeal region. Fgfr 1 transcripts were detected in all the cell types of the branchial arches (Fig. 1 A,B,C). Fgfr2 was co-expressed with Fgfr1 in this domain, but at the significantly lower level (Fig. 1 D,E,F). Expression of Fgfr3 was not detected in the presumptive second branchial arch region (Fig. $1 \mathrm{G}, \mathrm{H}, \mathrm{I}$ ), while in the first branchial arch Fgfr3 was detected at low level in all cell types. Coexpression of Fgfr1, Fgfr2 and Fgfr3 imply that they may have to some extent redundant roles in the branchial arch region.

Broad expression of Fgfr1 and Fgfr2 in the branchial arch region suggests that $F g f$ signaling specificity depends on the localized expression of $F g f$ ligands. We studied expression patterns of Fgf3, Fgf8, Fgf15, Fgf17 and Fgf18 genes in 7-9somite stage embryos. We did not detect expression of Fgf17 and Fgf18 in the pharyngeal region (data not shown). Fgf8 was

found to be broadly expressed in the ectoderm and endoderm of the branchial arch area (Fig. $2 \mathrm{G}, \mathrm{I}$ ). This observation is consistent with the previous studies of Fgf 8 expression pattern (Wall and Hogan, 1995). Fgf3 (Fig. 2A; Mahmood et al., 1996) and Fgf15 (Fig. 2C; McWhirter et al., 1997) were first detected at 8-somite stage in the pharyngeal region where their expression was found to be more restricted to the presumptive second arch area. Fgf3 was detected in neuroectoderm and surface ectoderm. Fgf3 expression in rhombomeres 4-6 continued in the surface ectodermal domain involving the otic placode and the presumptive second branchial arch region. Fgf15 was detected throughout the hindbrain except in the rhombomere 3. Fgf15 expression extended ventrally from the hindbrain toward the presumptive second branchial arch in both ectoderm and endoderm (Fig. 2E). Similar to Fgf3, surface ectoderm expression of Fgf15 included the otic placode and the presumptive second arch regions. Neither Fgf3 nor Fgf15 transcripts were detected in the mesenchymal cells. Spatial and temporal expression patterns of $F g f 3$ and Fgf15 suggest that they are important specifically for the second branchial arch formation and that there could be some functional redundancy between them.

We also analysed expression of the downstream targets of Fgfsignalling, Sprouty1 (Spry1) and Sprouty4 (Spry4), in 7-9-somite stage embryos. Whole mount in situ hybridization revealed broad expression of Spry1 and Spry4 in the pharyngeal region (Fig. 3 A,G; Minowada et al., 1999). Spry1 displayed graded expression with the highest expression at the level of rhombomere 4. To detect expression of Spry1 at the cellular level, whole mount in situ hybridization treated embryos were sectioned in transverse plane with a vibratome. Expression of Spry1 was found both in ectodermal, endodermal and mesenchymal cells of the branchial arches (Fig. 3 A,B,C). Spry4 was detected in
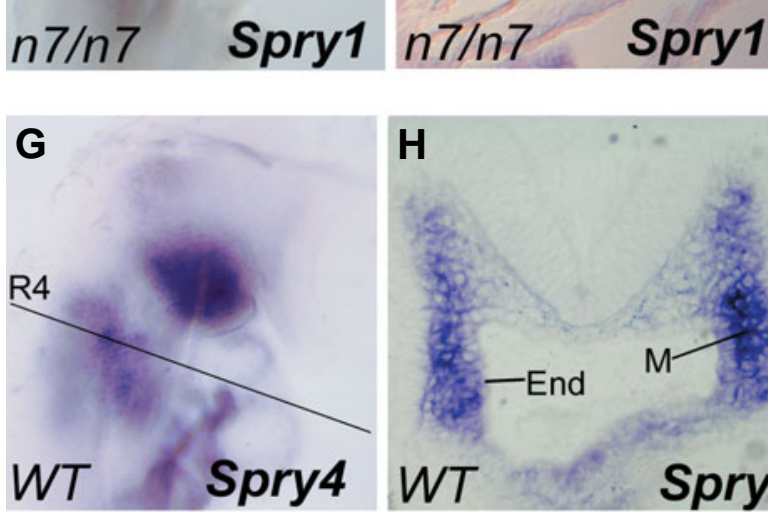

I

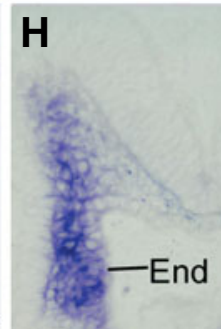

WT

J

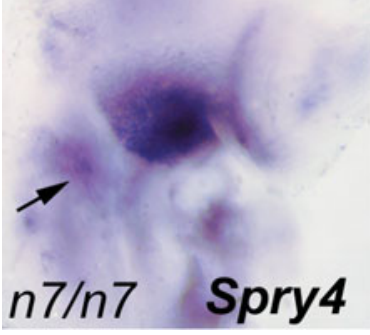

n7/n7 Spry4

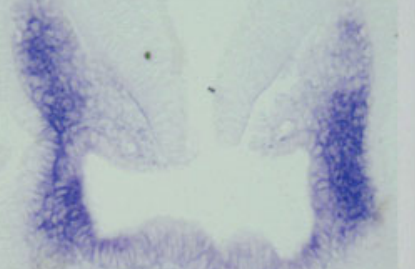

$n 7 / n 7$
Spry4 mesenchyme and at a lower level in the pharyngeal endoderm (Fig. 3 G,H). Expression patterns of Spry1 and Spry4 correlate with broad Fgf8 expression in the pharyngeal epithelium and suggest that FGF signalling is active in all the pharyngeal cell types.

Fgfr1 regulates gene expression in the epithelium of the presumptive second branchial arch

In the previous study we showed that Fgf3 expression in the pharyngeal ectoderm overlying the developing second branchial arch

Fig. 3. Expression patterns of Spry1 and Spry4 in the pharyngeal region of wild-type and Fgfr $^{\mathbf{n}}{ }^{\mathbf{7} / \mathbf{n 7}}$ embryos. Whole-mount RNA in situ hybridization at 7somite stage with Spry1 (A,D) and at 8-somite stage with Spry4 (G,I). Spry1 and Spry4 are expressed broadly in the pharyngeal region of control embryos $(\mathbf{A}, \mathbf{G})$. In mutants (D,I), pharyngeal expression of Spry1 and Spry4 appears normal in the first branchial arch but down-regulated posterior to it (indicated by arrows in

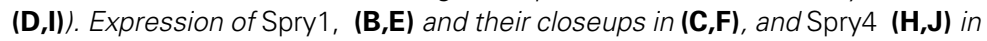
wild-type $(\mathbf{B}, \mathbf{C}, \mathbf{H})$ and mutant embryos $(\mathbf{E}, \mathbf{F}, \mathbf{J})$, was examined on transverse vibratome sections through the presumptive second arch (levels of sections are indicated in (A,E)). Note that only ectodermal expression of Spry1 is affected in Fgfr $1^{\mathrm{n} 7 / \mathrm{n} 7}$ embryos (indicated by arrowhead in $(\mathbf{E}, \mathbf{F})$ ). BA1, first branchial arch; Ect, ectoderm; End, endoderm; $H$, heart; $M$, mesenchyme; $R 4$, rhombomere 4. 

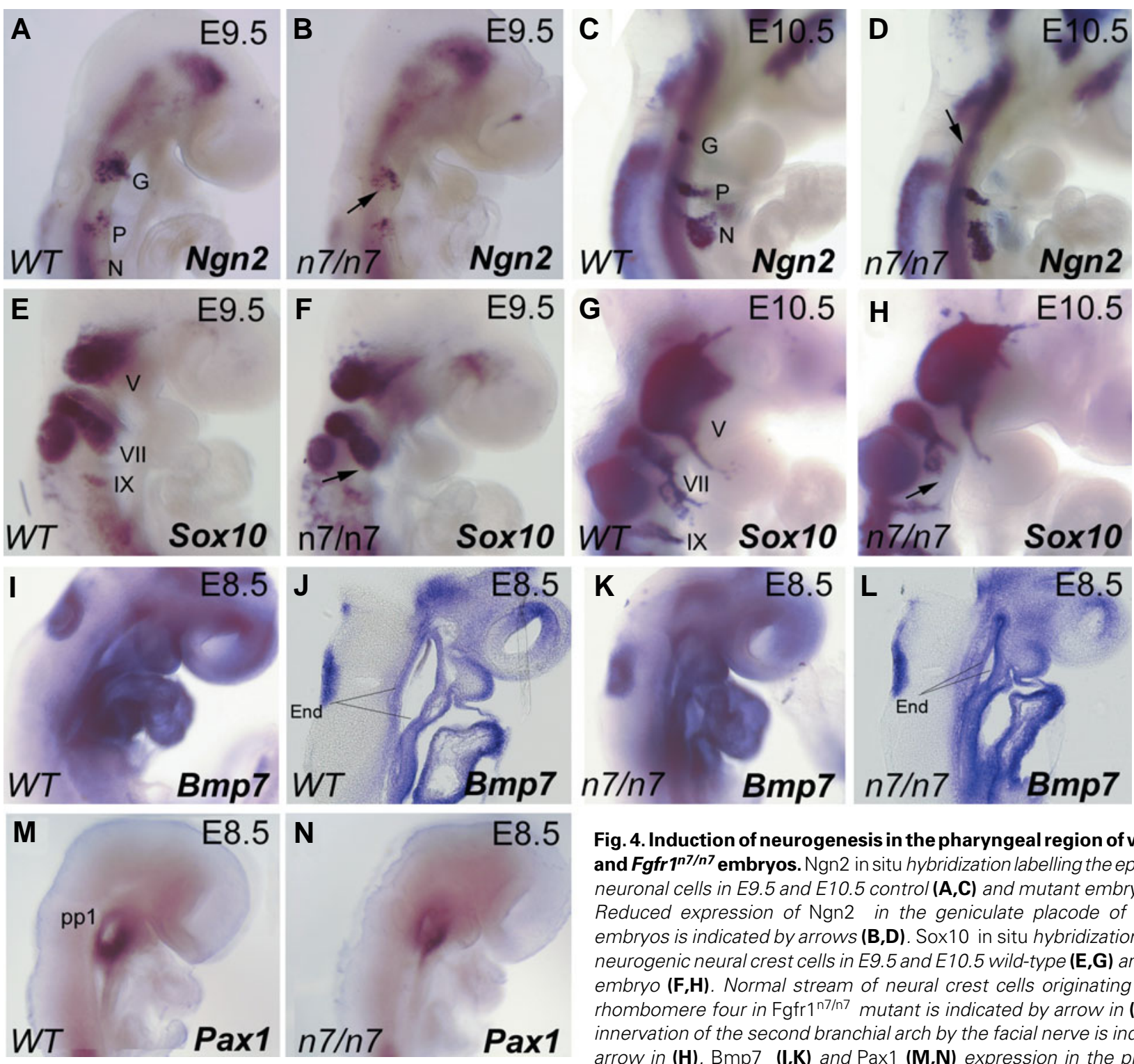

Fig. 4. Induction of neurogenesis in the pharyngeal region of wild-type and $\boldsymbol{F g f r}^{\mathbf{n} \mathbf{n} / \mathbf{n} \mathbf{7}}$ embryos. Ngn2 in situ hybridization labelling the epibranchial neuronal cells in E9.5 and E10.5 control (A,C) and mutant embryos (B,D) Reduced expression of $\mathrm{Ngn} 2$ in the geniculate placode of $\mathrm{Fgfr} 1^{\mathrm{n} 7 / \mathrm{n} 7}$ embryos is indicated by arrows (B,D). Sox10 in situ hybridization labelling neurogenic neural crest cells in E9.5 and E10.5 wild-type (E,G) and mutant embryo (F,H). Normal stream of neural crest cells originating from the rhombomere four in $\mathrm{Fgfr}^{\mathrm{n} 7 / \mathrm{n} 7}$ mutant is indicated by arrow in $(\mathbf{F})$. Failed innervation of the second branchial arch by the facial nerve is indicated by arrow in $\mathbf{( H )}$. Bmp7 $(\mathbf{I}, \mathbf{K})$ and $\operatorname{Pax} 1 \mathbf{(} \mathbf{M}, \mathbf{N})$ expression in the pharyngeal endoderm was detected by whole mount in situ hybridization in wild-type (I, $\mathbf{M})$ and mutant embryos $(\mathbf{K}, \mathbf{N})$ at 13-somite stage and 10-somite stage respectively. Expression of Bmp7, in wild-type (J) and mutant embryos (L) was examined on sagittal vibratome sections. BA2, second branchial arch; roman numbers indicate cranial nerves; End, endoderm.

was affected in embryos homozygous for a hypomorphic Fgfr In $^{7}$ allele, which expresses reduced levels of the Fgfr1 transcript (Partanen etal., 1998, Trokovic etal., 2003a). Down-regulation of Fgf3 was observed already at a stage prior to neural crest entry into the arch (Trokovic et al., 2003a). To confirm this result and to further understand the role of Fgfr1 in the branchial region, we analyzed additional pharyngeal epithelial markers in normal and Fgfr $y^{n 7 / n 7}$ embryos at 7-9-somite stage.

Fgf8 expression, detected by whole mount and section in situ hybridization (Fig 2. G-J), was unchanged in the Fgfr $1 n 7 / n 7 \mathrm{mu}-$ tants compared to control embryos. Similar to Fgf3 (Fig. 2 A,B; Trokovic et al., 2003a), Fgf15 expression was affected in the Fgfr $1^{n 7 / n 7}$ embryos compared to wild-type (Fig. 2C,D). Section in situhybridization revealed that ectodermal as well as endodermal expression is strongly down-regulated in mutant embryos com- pared with wild-type (Fig. 2 E,F).

In the Fgfr $1^{n 7 / n 7}$ embryos, expression of Spry 1 and Spry4 appeared normal in the first branchial arch, but was downregulated in the pharyngeal region around the presumptive second branchial arch, as shown by whole mount in situhybridisation (Fig. 3 A,D, G,I). Wild-type and Fgfr $177 / n 7$ embryos, labelled with Spry1 and Spry4, were sectioned in the transverse plane through the presumptive second branchial arch (Fig. 3 B,C,E,F,H,J). Already at 7-somite stage, strong reduction of Spry1 transcripts was detected in the ectoderm of the presumptive second branchial arch in the Fgfr ${ }^{17 / n 7}$ embryos compared with controls (Fig. $3 \mathrm{~B}, \mathrm{C}, \mathrm{E}, \mathrm{F})$. This was the earliest molecular change which we were able to detect in the Fgfr ${ }^{n 7 / n 7}$ embryos. Interestingly, expression of Spry1 (Fig. 3 B,C,E,F), as well as Spry4 (Fig. $3 \mathrm{H}, \mathrm{J}$ ), was detected both in the pharyngeal endoderm and mesenchyme of 

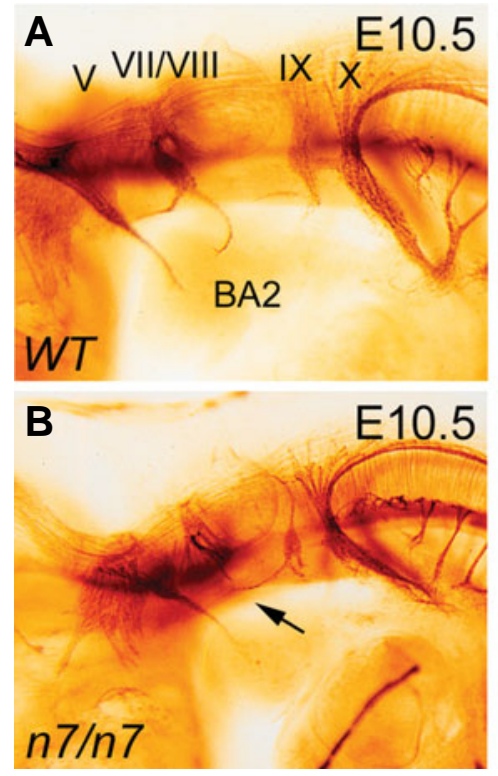
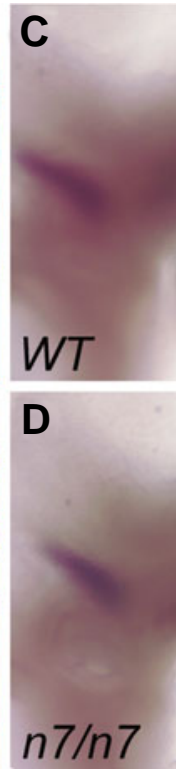

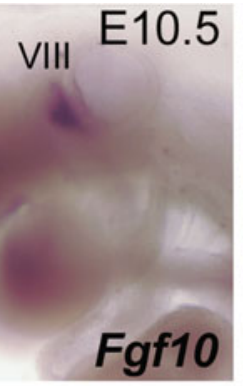

E10.5

Fgf10
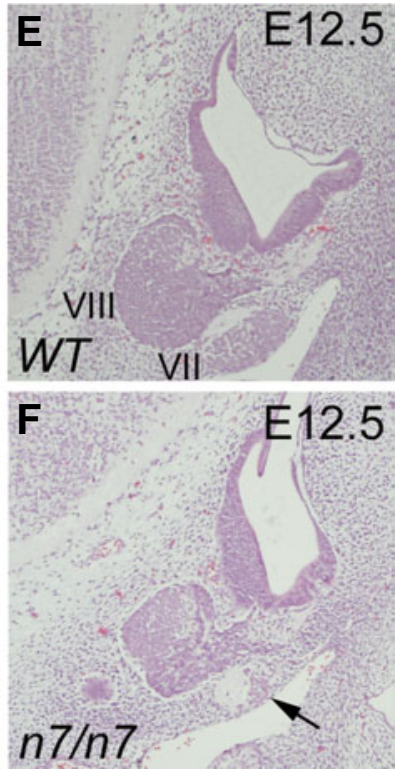

Fig. 5. Neuronal defects in Fgfr $1^{n 7 / n 7}$ embryos. Whole-mount antineurofilament stainings of E10.5 wildtype (A) and mutant (B) embryos. Defect in the VIlth cranial nerve of Fgfr $1 \mathrm{n} 7 / \mathrm{n} 7$ embryos is indicated by an arrow in (B). Whole mount in situ hybridisation detecting expression of Fgf10 in the VIIIth cranial ganglion of control (C) and Fgfr1n7/n7 (D) embryos at E10.5. Histological staining of E12.5 control (E) and Fgfr $1^{\mathrm{n} 7 / \mathrm{n} 7}$ (F) embryonic tissue sections at transverse plane. In the Fgfr $1^{n 7 / n 7}$ embryos, the VIIIth cranial ganglion appears normal while the VIIth ganglion is drastically reduced (arrow in (F)). BA2, second branchial arch; roman numbers indicate cranial nerves. the Fgfr $1^{n 7 / n 7}$ mutants. Molecular changes in the epithelium of the presumptive second branchial arch in the Fgfr $1 n 7 / n 7$ embryos imply that Fgfr1 is needed to establish appropriate patterns of gene expression in this domain.

\section{Impaired differentiation of the geniculate placode in Fgfr ${ }^{n 7 / n 7}$ embryos}

To further investigate the potential role of Fgfr1 in the pharyngeal ectoderm we analyzed its differentiation in the Fgfr $1^{n 7 / n 7}$ embryos. Surface ectoderm at distinct sites in the proximal region of the branchial aches gives rise to the epibranchial placodes and undergoes neurogenesis, revealed by Neurogenin 2 (Ngn2) expression (Sommer et al., 1996). We studied formation of the first (geniculate) epibranchial placode, which is related to the second branchial arch. We detected significantly lower number of Ngn2 positive cells in the geniculate placode of E9.5-10.5 Fgfr 1 n7/n7 embryos compared with control embryos (Fig. 4 A-D). The degree of $\mathrm{Ngn2}$ down-regulation correlated with the severity of the second branchial arch defect in the Fgfr ${ }^{n 7 / n 7}$ embryos. Expression of Ngn2 in the petrosal and nodose epibranchial placodes, related to the third and fourth branchial arches respectively, appeared normal in the mutants (Fig. 4 A,B,C,D).

Geniculate placode gives rise to neurons of the VIlth cranial ganglion together with neurogenic neural crest cells. To further

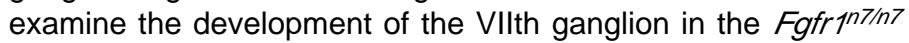
embryos, we looked at the expression of Sox10, an early marker of the neurogenic neural crest cells (Kuhlbrodt et al., 1998). We detected same pattern of Sox 10 expression in control and Fgfr $1^{n} 7 /$ $n 7$ embryos at E9.5 (Fig. 4 E,F). This result strongly suggested that deficient development of the geniculate placode and the VIlth ganglion (see below) is not caused by the primary defect in neural crest cells. At E10.5 Sox10 expression pattern in the Fgfr ${ }^{n 7 / n 7}$ embryos revealed failure of the facial nerve to innervate the second branchial arch (Fig. $4 \mathrm{G}, \mathrm{H}$ ). This defect probably reflects lack of normal cues in the second branchial arch of the Fgfr $1^{n 7 / n 7}$ mutants, which are needed for the neuronal innervation of the arch.

$B m p 7$ from the endoderm was shown to be the signal needed for induction of the epibranchial placodes in the ectoderm (Begbie et al., 1999). Thus, defect in the endoderm could lead to defect in formation of the geniculate placode observed in the Fgfr In $^{n / n 7}$ embryos. In order to address this possibility, we looked at the expression of Bmp7 in 13-somite stage control and mutant embryos (Fig. 4 I-L). We observed similar patterns of Bmp7 expression in wild-type and the Fgfr $1^{n / / n 7}$ embryos. Consistent with this we have detected Pax1 expression in the pharyngeal endoderm of both mutant and wild-type embryos at 10-somite stage (Fig. $4 \mathrm{M}, \mathrm{N}$ ). These results suggest that the placodal ectoderm in the Fgfr ${ }^{n>/ n 7}$ embryos receives inductive signal from the endoderm comparable to control embryos and that defect in geniculate placode formation is not caused by a defect in the endoderm.

Neural cells from the geniculate placode migrate inward and give rise to distal ganglia of VIlth cranial nerve, protruding their axonal processes toward rhombomere four and the second branchial arch (D'Amico-Martel and Noden, 1983). Using neurofilament staining at E10.5, we revealed deficient development of VIIth cranial nerve in Fgfr $1^{n 7 / n 7}$ embryos (Fig. 5 A,B), consistent with observed defect in formation of the geniculate placode. In the mutant embryos the VIlth cranial ganglion and its axonal projections were disorganized and reduced and bridging was seen between VIIth and IXth cranial nerves (arrow in Fig. 5 B).

The VIIIth cranial ganglion, derived from the otic vesicle, is positioned next to the VIIth ganglion. In order to distinguish between these two ganglia we have looked at Fgf10 expression (Pirvola etal., 2000). At E10.5, similar pattern of Fgf10 expression was detected in Fgfr $1^{n 7 / n 7}$ and wild-type embryos (Fig. 5 C,D). VIIlth ganglion appeared normal on the histological sections of E12.5 Fgfr $1^{n 7 / n 7}$ embryos, whereas the VIIth ganglion was drastically reduced (Fig. 5 E,F). Thus our results suggest that an ectodermal defect in the differentiation of the geniculate placode leads into abnormal development of the VIlth ganglion.

\section{Discussion}

FGF signalling appears to be important for multiple tissueinteractions during pharyngeal development, both within and be- 
tween different germ layers. Our results demonstrate that general reduction in the signalling intensity of one of the FGF receptors ( Fgfr 1 ) leads to a molecular defect in a specific domain of ectoderm overlying the presumptive second branchial arch. Signalling from this region appears to be important for development of both the second branchial arch and geniculate placode.

In the hypomorphic Fgfr $1^{n 7 / n 7}$ embryos, rhombomere 4 derived neural crest cells mostly fail to enter the second branchial arch. This defect is non-cell-autonomous, as a neural crest cell specific inactivation of Fgfr1 does not result in early second branchial arch defects, suggesting that Fgfr1 is providing permissive environment for the neural crest cell migration (Trokovic et al., 2003a). To address the primary target tissue requiring FGFR1 signalling, we analysed expression of known target genes of FGF signalling. We found that already at 8 -somite stage Fgfr $1^{n 7 / n 7}$ embryos display down-regulation of Spry1 in the ectoderm of the presumptive second branchial arch. This is the first defect observed in these mutants. Thereafter, pharyngeal epithelial markers Fgf15 (in both ectoderm and endoderm) and Fgf3 (in the ectoderm) are down-regulated within a narrow window of time. We propose that Fgfr1 is important for expression of the ectodermal signalling molecules and consequently for the normal patterning of other branchial arch components. We can not completely rule out the possibility that the changes in the ectodermal gene-expression in the Fgfr $1^{n / / n 7}$ mutants are caused by a defect in another tissue, for example endoderm. However, the normal level of Pax 1 and $B m p 7$ transcripts in the pharyngeal endoderm of the Fgfr $1^{n 7 / n 7}$ mutants at E8.5 suggest that the endoderm is correctly patterned in the hypomorphic Fgfr 1 mutants at this stage. Ectoderm and endoderm -specific Fgfr1 inactivation can be expected to provide a definitive answer to the question of target tissue specificity.

In addition to changes in the gene-expression, we demonstrated that this specific domain of ectoderm fails to differentiate normally in Fgfr $1^{n 7 / n 7}$ embryos. Cranial nerves have heterogeneous origin, arising from both neural crest cells and ectodermal placodes. In the Fgfr $1^{n 7 / n 7}$ embryos development of the VIIth cranial nerve is affected. We observed fewer $\mathrm{Ngn} 2$ expressing cells in the geniculate placode of the Fgfr $1^{n 7 / n 7}$ embryos, whereas expression of Sox10, marking the neuronal crest cells, was normal at E9.5. These results imply that cranial nerve defects in the Fgfr $1^{n 7 / n 7}$ mutants are initiated in its ectodermal component.

Pharyngeal endoderm has been shown to be the source of the inductive signal $B m p 7$, which is necessary and sufficient for induction of neurogenesis in epibranchial placodes (Begbie et al., 1999). In the Fgfr $1^{n 7 / n 7}$ embryos $B m p 7$ is normally expressed in the pharyngeal endoderm. Thus, we suggest that Fgfr1 is required in the surface ectoderm of the future second arch well before neurogenesis in the geniculate placode is initiated by $B m p 7$ from the pharyngeal endodem.

What could be the FGF ligand activating FGFR1 in the ectoderm? Based on the expression analyses performed by us and others, FGF8 appears to be one possible candidate. Fgf8 is expressed both in pharyngeal ectoderm and endoderm and FGF8 could thus activate FGFR1 in either autocrine or paracrine fashion. However, Fgf8 expression is widespread in the pharyngeal region. Therefore, additional factors must contribute to the localized activation of $F g f 3$ and $F g f 15$ expression in the ectoderm overlying the presumptive second branchial arch
In summary, our results shed light on the molecular basis of intercellular interactions in the pharyngeal region (Fig. 6). We show that signalling through Fgfr1 is needed for establishment of a local signalling center revealed by Fgf3 and Fgf15 expression. Perhaps similar to other secondary organizing centers involving FGFs, such as the isthmus, the anterior neural ridge and hindbrain (Marin and Charnay, 2000), we propose organizing role for
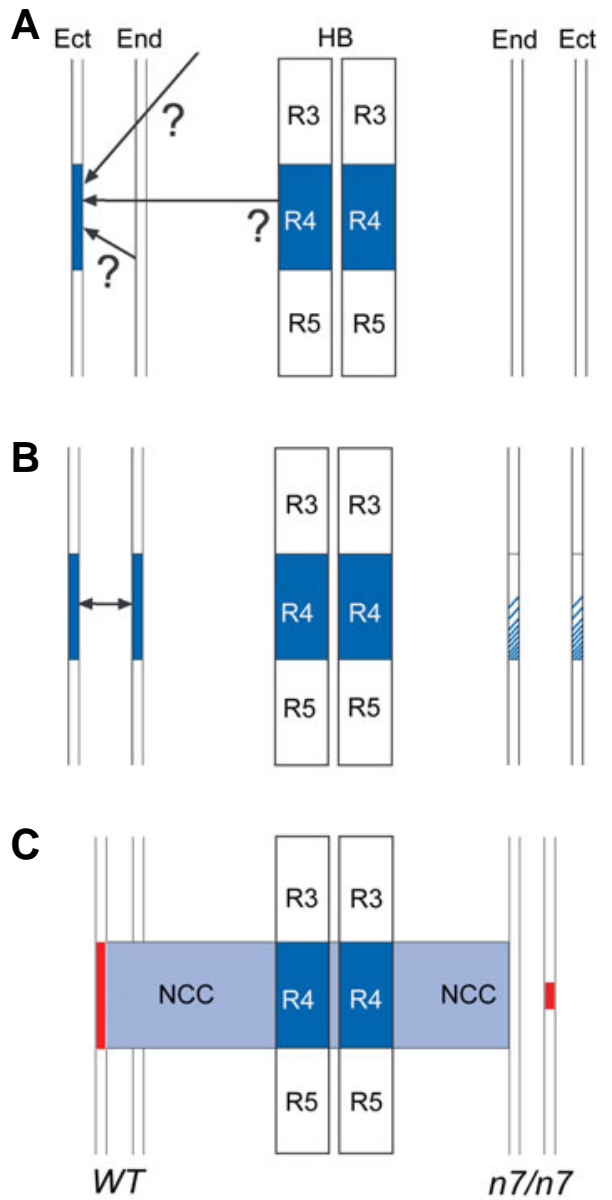

Fig. 6. Proposed model for the function of FGFR1 in regional specification of the pharyngeal ectoderm. In wild-type embryos lon the left side of schematic presentations in (A,B,C)) FGF signalling through FGFR1 regulates gene expression in the surface ectoderm overlying the presumptive second branchial arch (dark blue in (A)). It is possible that the FGFs are secreted from the rhombomere four in the hindbrain, the pharyngeal endoderm, or some other source lquestion marks in (A)). Subsequent interactions between pharyngeal ectoderm and endoderm (dark blue in (B)) ensure proper integration of the second branchial arch cell types (neural crest cells migration into the second branchial arch indicated in light blue in (C)) and formation of the geniculate placode (in red). In the $\mathrm{Fgfr}^{\mathrm{n} 7 / \mathrm{n} 7}$ mutants (on the right side of the schematic presentations in (A, $\mathbf{B}, \mathbf{C})$ ), gene expression in the ectoderm overlying the presumptive second branchial arch is deficient (A). As a result, proper signalling between ectoderm and endoderm fails. This leads to defects in tissue integration and differentiation (C) including fusion of the first and the second pharyngeal pouch, failure of neural crest cells to migrate into the second branchial arch and deficient geniculate epibranchial placode. BA2, second branchial arch; Ect, ectoderm; End, endoderm; NCC, neural crest cells; R35, rhombomere three-five. 
FGF signalling in the ectoderm covering the presumptive second arch. However, in contrast to many of the well-studied secondary organizers this ectodermal domain appears to be only a transient source of signals, Fgf3 and Fgf15, being expressed only at 8-12somite stage. Interestingly, this domain appears to be at the same axial level as rhombomere 4, which has been shown to be a source of FGF signals patterning the surrounding rhombomeres (Maves et al., 2002, Walshe et al., 2002). Rhombomere 4 might also induce Fgf3 and Fgf15 expression in the overlaying ectoderm, but the other tissues are possible sources of such signals as well (Fig. 6A). In addition to the ectoderm itself, this signalling center in the pharyngeal ectoderm may have impact also on other cell types, including neural crest, pharyngeal endoderm as well as aortic arches. We suggest that defective development and integration of distinct tissues in the second branchial arch region (failure of neural crest cells to populate the second arch, fusion of the first and the second pharyngeal pouch, disintegrated second aortic arch, deficient geniculate epibranchial placode and VIIth cranial nerve) are probably all related to the defect in the pharyngeal ectodermal signalling center in the Fgfr $1^{n / / n 7}$ mutants.

\section{Materials and Methods}

\section{Embryos}

Analyses of mice carrying hypomorphic Fgfr1 allele ( Fgfr $1^{n 7}$; Partanen et al., 1998) were carried out in outbred (ICR) background. Embryonic age was estimated by counting somites. Wild-type allele was detected by upstream 5'-CCCCATCCCATTTCCTTACCT-3' and downstream 5'TTCTGGTGTGTCTGAAAACAGCT-3' oligonucleotide primers (145 bp product). Fgfr $1^{n 7}$ allele was detected by upstream 5'AATAGGTCCCTCGACGGTATC-3' and downstream 3'CTGGGTCAGTGTGGACAGTGT-5' primers (166 bp product).

Embryos for in situhybridization were fixed in $4 \%$ paraformaldehyde in phosphate buffered saline (PBS) at $4^{\circ} \mathrm{C}$ overnight.

\section{In situ hybridization}

Whole-mount RNA in situhybridization was performed as described by (Henrique et al., 1995).

For vibratome sectioning stained embryos were embedded into gelatine-albumin ( $0.45 \%$ gelatin, $25 \%$ albumin, $20 \%$ sucrose) fixed with $2.5 \%$ glutaraldehyde and cut at $20 \mu \mathrm{m}$ in the transverse plane.

In situ hybridisation on paraffin-embedded tissue sections, cut at $7 \mu \mathrm{m}$, was performed using $35 \mathrm{~S}$ labelled riboprobes as described (Wilkinson and Green 1990).

Antisense riboprobes were as follows: Fgfr1 (Trokovic et al., 2003b), Fgfr2 (a gift from Alka Mansukhani), Fgfr3 (Peters et al., 1993), Fgf3 (Wilkinson etal., 1988), Fgf8 (Crossley and Martin, 1995), Fgf10 (Bellusci et al., 1997), Fgf15 (McWhirter et al., 1997), Spry1 and Spry4 (gift from Seppo Vainio), Pax1 (IMAGE 1327502), Erm (IMAGE 3674281), Bmp7 (IMAGE 5121825), Sox10 (IMAGE 4165363) and Ngn2 (IMAGE 2922473).

On average five and at least three mutants and same number of littermate controls were hybridized with each probe.

\section{Anti-neurofilament immunohistochemistry}

Whole mount neurofilament staining with monoclonal anti-neurofilament antibody (Sigma N-5139) was performed according to combination of procedures by (Dent et al., 1989; LeMotte et al., 1989).

\section{Acknowledgements}

We thank Eija Koivunen and Päivi Hannuksela for expert technical assistance. This work was supported by the Academy of Finland, the Sigrid Juselius foundation, Biocentrum Helsinki and Viikki Graduate School in
Biosciences (N. T., R.T.).

\section{References}

BEE, J. and THOROGOOD, P. (1980). The role of tissue interactions in the skeletogenic differentiation of avian neural crest cells. Dev. Biol 78: 47-62.

BEGBIE, J., BRUNET, J.F., RUBENSTEIN, J.L. and GRAHAM, A. (1999). Induction of the epibranchial placodes. Dev. 126: 895-902.

BELLUSCI, S., GRINDLEY, J., EMOTO, H., ITOH, N. and HOGAN, B.L. (1997). Fibroblast growth factor 10 (FGF10) and branching morphogenesis in the embryonic mouse lung. Dev. 124, 4867-78.

COULY G. and LE DOUARIN N.M. (1990). Head morphogenesis in embryonic avian chimeras: evidence for a segmental pattern in the ectoderm corresponding to the neuromeres. Dev. 108: 543-558.

COULY, G., CREUZET, S., BENNACEUR, S., VINCENT, C. and LE DOUARIN, NM. (2002). Interactions between Hox-negative cephalic neural crest cells and the foregut endoderm in patterning the facial skeleton in the vertebrate head. Dev. 129: 1061-1073.

CROSSLEY, P.H. and MARTIN, G.R. (1995). The mouse Fgf8 gene encodes a family of polypeptides and is expressed in regions that direct outgrowth and patterning in the developing embryo. Dev. 121: 439-451.

D'AMICO-MARTEL, A. and NODEN, D.M. (1983). Contributions of placodal and neural crest cells to avian cranial peripheral ganglia. Am. J. Anat. 166: 445-468.

DAVID, N.B., SAINT-ETIENNE, L., TSANG, M., SCHILLING, T.F. and ROSA, F.M. (2002). Requirement for endoderm and FGF3 in ventral head skeleton formation. Dev. 129: 4457-4468.

DENG, C.X., WYNSHAW-BORIS, A., SHEN, M.M., DAUGHERTY, C., ORNITZ, D.M. and LEDER, P. (1994). Murine FGFR-1 is required for early postimplantation growth and axial organization. Genes Dev. 8: 3045-3057.

DENT, J.A., POLSON, A.G. and KLYMKOWSKY, M.W. (1989). A whole-mount immunocytochemical analysis of the expression of the intermediate filament protein vimentin in Xenopus. Dev. 105: 61-74.

GAVALAS, A., TRAINOR, P., ARIZA-MCNAUGHTON, L. and KRUMLAUF, R. (2001). Synergy between Hoxa1 and Hoxb1: the relationship between arch patterning and the generation of cranial neural crest. Dev. 128: 3017-3027.

GOLDING, J.P., TRAINOR, P., KRUMLAUF, R. and GASSMANN, M. (2000). Defects in pathfinding by cranial neural crest cells in mice lacking the neuregulin receptor ErbB4. Nat. Cell Biol. 2: 103-109.

GOLDING, J.P., DIXON, M. and GASSMANN, M. (2002). Cues from neuroepithelium and surface ectoderm maintain neural crest-free regions within cranial mesenchyme of the developing chick. Dev. 129: 1095-1105.

GRAHAM, A. and SMITH, A. (2001). Patterning the pharyngeal arches. Bioessays 23: 54-61.

HENRIQUE, D., ADAM, J., MYAT, A., CHITNIS, A., LEWIS, J. and ISH-HOROWICZ, D. (1995). Expression of a Delta homologue in prospective neurons in the chick. Nature 375: 787-790.

HUNT, P., WHITING, J., MUCHAMORE, I., MARSHALL, H. and KRUMLAUF, R. (1991). Homeobox genes and models for patterning the hindbrain and branchial arches. Dev. Suppl. 1: 187-196.

KAUFMAN, M.H. and BARD, J.B.L. (1999). The anatomical basis of mouse development, Academic press, San Diego.

KONTGES, G. and LUMSDEN, A. (1996). Rhombencephalic neural crest segmentation is preserved throughout craniofacial ontogeny. Dev. 122: 3229-3242.

KUHLBRODT, K., HERBARTH, B., SOCK, E., HERMANS-BORGMEYER, I. and WEGNER M. (1998). Sox10, a novel transcriptional modulator in glial cells. J. Neurosci. 18: 237-250.

LEMOTTE, P.K., KUROIWA, A., FESSLER, L.I. and GEHRING, W.J. (1989). The homeotic gene Sex Combs Reduced of Drosophila: gene structure and embryonic expression. EMBO J. 8: 219-227.

LUMSDEN, A., SPRAWSON, N. and GRAHAM, A. (1991). Segmental origin and migration of neural crest cells in the hindbrain region of the chick embryo. Dev.113: 1281-1291.

MAHMOOD, R., MASON, I.J. and MORRISS-KAY, G.M. (1996). Expression of Fgf3 in relation to hindbrain segmentation, otic pit position and pharyngeal arch 
morphology in normal and retinoic acid- exposed mouse embryos. Anat Embryol (Berl) 194: 13-22.

MARIN, F. and CHARNAY, P. (2000). Hindbrain patterning: FGFs regulate Krox20 and $\mathrm{mafB} / \mathrm{kr}$ expression in the otic/preotic region. Dev. 127: 4925-4935.

MAVES, L., JACKMAN, W. and KIMMEL, C.B. (2002). FGF3 and FGF8 mediate a rhombomere 4 signaling activity in the zebrafish hindbrain. Dev. 129: 3825-3837.

MCWHIRTER, J.R., GOULDING, M., WEINER, J.A., CHUN, J. and MURRE, C. (1997). A novel fibroblast growth factor gene expressed in the developing nervous system is a downstream target of the chimeric homeodomain oncoprotein E2APbx1. Dev. 124: 3221-3232.

MEIER, S. and TAM, P.P. (1982). Metameric pattern development in the embryonic axis of the mouse. I. Differentiation of the cranial segments. Differentiation 21: 95108.

MINOWADA, G., JARVIS, L.A., CHI, C.L., NEUBUSER, A., SUN, X., HACOHEN, N., KRASNOW, M.A. and MARTIN, G.R. (1999). Vertebrate Sprouty genes are induced by FGF signaling and can cause chondrodysplasia when overexpressed. Dev. 126: 4465-4475.

NODEN, D.M. (1983). The role of the neural crest in patterning of avian cranial skeletal, connective and muscle tissues. Dev. Biol. 96: 144-165.

NODEN, D.M. (1986). Patterning of avian craniofacial muscles. Dev. Biol. 116: 347356.

NODEN, D.M. (1988). Interactions and fates of avian craniofacial mesenchyme. Dev. Suppl. 103: 121-140.

PARTANEN, J., SCHWARTZ, L. and ROSSANT, J. (1998). Opposite phenotypes of hypomorphic and $\mathrm{Y} 766$ phosphorylation site mutations reveal a function for Fgfr1 in anteroposterior patterning of mouse embryos. Genes Dev. 12: 2332-2344.

PETERS, K., ORNITZ, D., WERNER, S. and WILLIAMS, L. (1993). Unique expression pattern of the FGF receptor 3 gene during mouse organogenesis. Dev. Biol. 155: 423-430.

PIOTROWSKI, T. and NUSSLEIN-VOLHARD, C. (2000). The endoderm plays an important role in patterning the segmented pharyngeal region in zebrafish (Danio rerio). Dev. Biol. 225: 339-356.

PIRVOLA, U., SPENCER-DENE, B., XING-QUN, L., KETTUNEN, P., THESLEFF, I., FRITZSCH, B., DICKSON, C. and YLIKOSKI, J. (2000). FGF/FGFR-2(IIIb) signaling is essential for inner ear morphogenesis. J. Neurosci. 20: 6125-6134.

RUHIN, B.C. (2003). Patterning of the hyoid cartilage depends upon signals arising from the ventral foregut endoderm. Dev. Dyn. 228: 239-246.

SCHILLING, T.F., PRINCE, V. and INGHAM, P.W. (2001). Plasticity in zebrafish hox expression in the hindbrain and cranial neural crest. Dev. Biol. (N.Y.1985.) 231: 201-216.

SERBEDZIJA, G.N., BRONNER-FRASER, M. and FRASER, S.E. (1992). Vital dye analysis of cranial neural crest cell migration in the mouse embryo. Dev. 116: 297307.

SHIGETANI, Y., NOBUSADA, Y. and KURATANI, S. (2000). Ectodermally derived FGF8 defines the maxillomandibular region in the early chick embryo: epithelialmesenchymal interactions in the specification of the craniofacial ectomesenchyme. Dev. Biol. 228: 73-85.

SOMMER, L., MA, Q. and ANDERSON, D.J. (1996). Neurogenins, a novel family of atonal-related bHLH transcription factors, are putative mammalian neuronal determination genes that reveal progenitor cell heterogeneity in the developing
CNS and PNS. Mol. Cell Neurosci. 8: 221-241.

TRAINOR, P.A., TAN, S.S. and TAM, P.P. (1994). Cranial paraxial mesoderm: regionalisation of cell fate and impact on craniofacial development in mouse embryos. Dev. 120: 2397-2408.

TRAINOR, P. and KRUMLAUF, R. (2000). Plasticity in mouse neural crest cells reveals a new patterning role for cranial mesoderm. Nat. Cell Biol. 2: 96-102.

TRAINOR, P.A. and KRUMLAUF R. (2001). Hox genes, neural crest cells and branchial arch patterning. Curr. Opin. Cell Biol. 13: 698-705.

TRAINOR, P.A., SOBIESZCZUK, D., WILKINSON, D. and KRUMLAUF, R. (2002a). Signalling between the hindbrain and paraxial tissues dictates neural crest migration pathways. Dev. 129: 433-442.

TRAINOR, P.A., ARIZA-MCNAUGHTON, L. and KRUMLAUF, R. (2002b). Role of the isthmus and FGFs in resolving the paradox of neural crest plasticity and prepatterning. Science 295: 1288-1291.

TROKOVIC, N., TROKOVIC, R., MAI, P. and PARTANEN, J. (2003a). Fgfr1 regulates patterning of the pharyngeal region. Genes Dev. 17: 141-153.

TROKOVIC, R., TROKOVIC, N., HERNESNIEMI, S., PIRVOLA, U., VOGT, W.D., ROSSANT, J., MCMAHON, A.P., WURST, W. and PARTANEN, J. (2003b). FGFR1 is independently required in both developing mid- and hindbrain for sustained response to isthmic signals. EMBO J. 22: 1811-1823.

TUCKER, A.S. and SHARPE, P.T. (1999). Molecular genetics of tooth morphogenesis and patterning: the right shape in the right place. J. Dental Res. 78: 826-834.

VEITCH, E., BEGBIE, J., SCHILLING, T.F., SMITH, M.M. and GRAHAM, A. (1999). Pharyngeal arch patterning in the absence of neural crest. Curr. Biol. 9: 14811484.

WALL, N.A. and HOGAN, B.L. (1995). Expression of bone morphogenetic protein-4 (BMP-4), bone morphogenetic protein-7 (BMP-7), fibroblast growth factor-8 (FGF8 ) and sonic hedgehog $(\mathrm{SHH})$ during branchial arch development in the chick. Mech. Dev. 53: 383-392.

WALSHE, J., MAROON, H., MCGONNELL, I.M., DICKSON, C. and MASON, I. (2002). Establishment of hindbrain segmental identity requires signaling by FGF3 and FGF8. Curr. Biol. 12: 1117-1123.

WASKIEWICZ, A.J., RIKHOF, H.A. and MOENS, C.B. (2002). Eliminating zebrafish pbx proteins reveals a hindbrain ground state. Developmental Cell 3: 723-733.

WILKINSON, D.G., PETERS, G., DICKSON, C. and MCMAHON, A.P. (1988). Expression of the FGF-related proto-oncogene int-2 during gastrulation and neurulation in the mouse. EMBO J. 7: 691-695.

WILKINSON, D.G. and GREEN, J. (1990). In situ hybridization and the threedimensional construction of serial sections. In: Copp A.J., Cockroft D.L., editors. Postimplatation Mammalian Embryos, Oxford University Press, Oxford U., pp. 155-171.

YAMAGUCHI, T.P., HARPAL, K., HENKEMEYER, M. and ROSSANT, J. (1994). Fgfr1 is required for embryonic growth and mesodermal patterning during mouse gastrulation. Genes. Dev. 8: 3032-3044.

Received: February 2005

Reviewed by Referees: May 2005

Modified by Authors and Accepted for Publication: July 2005 